\title{
BMJ Open Implementation of an algorithm for the identification of breast cancer deaths in German health insurance claims data: a validation study based on a record linkage with administrative mortality data
}

Ingo Langner, ${ }^{1}$ Christoph Ohlmeier, ${ }^{2}$ Ulrike Haug, ${ }^{1,3}$ Hans Werner Hense, ${ }^{4,5}$ Jonas Czwikla, ${ }^{\oplus, 6}$ Hajo Zeeb ${ }^{1,3}$

To cite: Langner I, Ohlmeier C, Haug U, et al. Implementation of an algorithm for the identification of breast cancer deaths in German health insurance claims data: a validation study based on a record linkage with administrative mortality data. BMJ Open 2019;9:e026834. doi:10.1136/ bmjopen-2018-026834

- Prepublication history and additional material for this paper are available online. To view these files, please visit the journal online (http://dx.doi. org/10.1136/bmjopen-2018026834).

Received 21 September 2018

Revised 28 May 2019

Accepted 2 July 2019

D Check for updates

(C) Author(s) (or their employer(s)) 2019. Re-use permitted under CC BY-NC. No commercial re-use. See rights and permissions. Published by BMJ.

For numbered affiliations see end of article.

Correspondence to

Dr Ingo Langner;

langner@leibniz-bips.de

\section{ABSTRACT}

Objective To adapt a Canadian algorithm for the identification of female cases of breast cancer (BC) deaths to German health insurance claims data and to test and validate the algorithm by comparing results with official cause of death $(\mathrm{CoD})$ data on the individual and the population level.

Design Validation study, secondary data, medical claims. Setting Claims data of two statutory health insurance providers (SHIs) for inpatient and outpatient care, CoD added via record linkage with epidemiological cancer registry (ECR). Participants All women insured with the two SHIs and who deceased in the period 2006-2013, were residents of North Rhine Westphalia (NRW) and were linked with ECR data: $n=22413$.

Main outcome measures Based on inpatient and outpatient diagnoses in the year before death, six algorithms were derived and the accordance of the algorithm-based $\mathrm{CoD}$ with the official $\mathrm{CoD}$ was evaluated calculating specificity, sensitivity, negative and positive predictive values (NPV, PPV). Furthermore, algorithmbased age-specific BC mortality rates covering several calendar years were calculated for the entire insured female population and compared with official national rates.

Results Our final algorithm, derived from the NRW subsample, comprised codes indicating the presence of $\mathrm{BC}$, metastases, a terminal illness phase and the absence of codes for other tumours. Overall, specificity, sensitivity, NPV and PPV of this algorithm were 97.4\%, 91.3\%, 98.9\% and $81.7 \%$, respectively. In the age range $40-80$ years, sensitivity and PPV slightly decreased with increasing age. Algorithm-based age-specific BC mortality rates agreed well with official rates except for the age group 85 years and older.

Conclusions The algorithm-based identification of $\mathrm{BC}$ deaths in German claims data is feasible and valid, except for higher ages. The algorithm to ascertain BC mortality rates in an epidemiological study seems applicable when information on the official $\mathrm{CoD}$ is not available in the original database.

\section{Strengths and limitations of this study}

- This is the first study evaluating whether an algorithm for the identification of breast cancer deaths in Canadian claims data can be adapted to German health insurance claims data.

- Causes of death classifications of different algorithm versions were directly compared with the official cause of death on the individual level and indirectly compared with official vital statistics on the population level.

- The sample for testing and validating the algorithm on the individual level was based on a high-quality record linkage and included 22413 women who died in the period 2006-2013.

- The test and validation sample was restricted to the age range $40-80$ years.

- The study results are based on German claims data, however, the procedures are considered transferable to other settings with some adaptation.

\section{INTRODUCTION}

Cause-specific mortality is a major outcome in epidemiological cohort studies. Especially for studies with rare outcomes, where a great amount of cumulative follow-up years with a consistent exposure ascertainment is essential, claims data constitute a promising data source. However, in German claims data, the official cause of death $(\mathrm{CoD})$ is not included and individual record linkage with data sources providing this information is limited due to data protection regulations. Such record linkage is most likely feasible only on a regional level due to the regional character of suitable registries comprising $\mathrm{CoD}$ information which, however, restricts the usable study population. 
For the intended nationwide monitoring and evaluation of the impact of the German mammography screening programme (MSP) ${ }^{1}$ on breast cancer (BC) mortality, ${ }^{2}$ claims data as included in the German Pharmacoepidemiological Research Database (GePaRD) represent one of the most suitable data sources. The data comprise demographic information, inpatient and outpatient diagnoses, health services, and detailed information concerning the participation in the MSP. Further, deceased individuals can be identified in GePaRD because 'death' is coded either as the reason for the end of insurance coverage or for the discharge from hospital. The lack of information on the official CoD, however, represents a limitation of this data source.

As CoD information is essential for epidemiological cohort studies such as the intended nationwide monitoring and evaluation of BC mortality in the MSP, there is a need to add at least the information 'death due to BC (yes/no)'. In order to mimic official $\mathrm{CoD}$ information, we, therefore, aimed to implement, optimise and validate a claims data-based algorithm for the identification of $\mathrm{BC}$ deaths among deceased females in GePaRD, inspired by the approach developed by Gagnon $e t a \hat{l}$ for Canadian administrative data.

\section{METHODS}

\section{Data source}

Analyses were based on the GePaRD database, which has been described elsewhere. ${ }^{4-6}$ In brief, GePaRD includes pseudonymised claims data from four statutory health insurance providers (SHIs) and contains information on about 20 million individuals from all over Germany who have been insured at one of the participating SHIs since 2004 or later. The database contains information on demographic characteristics, the start and end of insurance periods, hospital stays, outpatient physician visits and outpatient prescriptions. The hospital data comprise information on the dates of admission and discharge, admission diagnoses, one main discharge diagnosis (which specifies the disease causing the hospital stay), further main and secondary hospital diagnoses, diagnostic and therapeutic procedures with their respective dates, as well as the reason for hospital discharge (eg, 'treatment terminated regularly', 'transfer to another hospital', 'deceased' and others). Outpatient data contain information on outpatient diagnoses, treatments and procedures. Outpatient diagnoses are recorded per quarter and are distinguishable into different types, for example, 'confirmed'. Diagnoses are coded according to the German modification of the International Classification of Diseases, 10th Revision (ICD-10-GM). Information related to the death of individuals can be obtained from the variable specifying the reason for the end of insurance (eg, "change to another insurance company', 'deceased' and others) and for subjects dying in hospital also from the respective variable specifying the cause of the hospital discharge (about $50 \%$ of all deaths in GePaRD).

The algorithm-based individual assignment of a death by $\mathrm{BC}$ in $\mathrm{GePaRD}$ was compared with the individual official CoD provided by the State Cancer Registry of North Rhine Westphalia (CR-NRW) that holds information on the official causes of each deceased person in NRW since 2006. This information was linked via a probabilistic record linkage to the claims data on the person level (for details see reference ${ }^{7}$ ). In addition, to assess the performance of the algorithm-based classification on the population level, age-specific $\mathrm{BC}$ death rates estimated in the entire GePaRD data base were compared with data from the German Centre for Cancer Registry Data (ZfKD) that provided data on the national $\mathrm{BC}$ mortality rates.

\section{Patient and public involvement}

Patients or public were not involved.

\section{Study populations}

Individual-level analysis

All death cases occurring between 2006 and 2013 among female GePaRD residents of NRW (as identified in GePaRD) who had been continuously insured in the year before death and whose GePaRD data could be successfully linked with the CR-NRW records, formed the study population for the algorithm validation on the individual level (see also online supplementary data 1). Data year restrictions were due to limitations imposed by legal authorities. The age range of women included in this subsample of GePaRD was limited to 40-80years encompassing the age range of eligibility for the MSP (50-69 years) and the adjacent decades.

\section{Population-level analysis}

For the calculation of claims data-based age-specific BC mortality rates at the population level, all women in GePaRD irrespective of place of residence and age insured in 2007, 2010 and 2012, respectively, were included in the study population. Deceased women were identified via a corresponding data entry on the reason for the end of insurance coverage or for the discharge from hospital. The date of death was defined accordingly as the date of the end of insurance or the date of hospital discharge.

\section{Algorithm for the identification of BC deaths}

Based on Canadian routine health data, Gagnon $e t a \hat{l}$ developed an algorithm for the identification of women who had died of BC. The identification was based on ICD-9-coded inpatient diagnoses from all hospital stays of a single person ever recorded at provincial hospital discharge databases. Gagnon et al applied three criteria to identify BC as the CoD. First, women had to be diagnosed with BC (ICD-9: 174.0-174.9) with or without regional metastases (ICD-9: 196.0-196.9). Second, remote metastases had to be documented (ICD-9: 197.0-199.0). Third, the respective women had to have diagnoses indicating terminal illness (eg, septicaemia, pathological fractures or gastrointestinal haemorrhage; for details see reference ${ }^{3}$ ).

To adapt the algorithm for use in German claims data, the diagnostic criteria were implemented according to the ICD-10-GM. We considered a 1-year period before death to evaluate the criteria for the identification of BC deaths. 
As in the Canadian approach, we considered information from hospitalisations. Furthermore, in extension of Gagnon et $a l l^{3}$ we also considered outpatient diagnoses and differentiated between hospital main discharge diagnoses and hospital secondary diagnoses. The latter facilitated further options to adapt the algorithm. The criteria used by Gagnon $e t a \hat{l}$ were expanded by information on comorbidity. Overall, we ended up with eight criteria (Cr1-8) (online supplementary data 2). Cr1-5 were set a priori in which Cr1-4 with the intention to mimic the algorithm criteria of Gagnon et al. ${ }^{3}$ Cr6-8 were set after additional considerations following a descriptive analysis of the official causes of death of those cases selected as false positives by algorithm version $\mathrm{C}$ to evaluate which were the most prominent groups of cancer causes of death among these cases. Different combination of all criteria resulted in six different versions of the algorithm (A-F) as shown in figure 1 .

\section{Statistical analyses}

To evaluate the performance of each algorithm, we randomly divided the subsample for individual-level

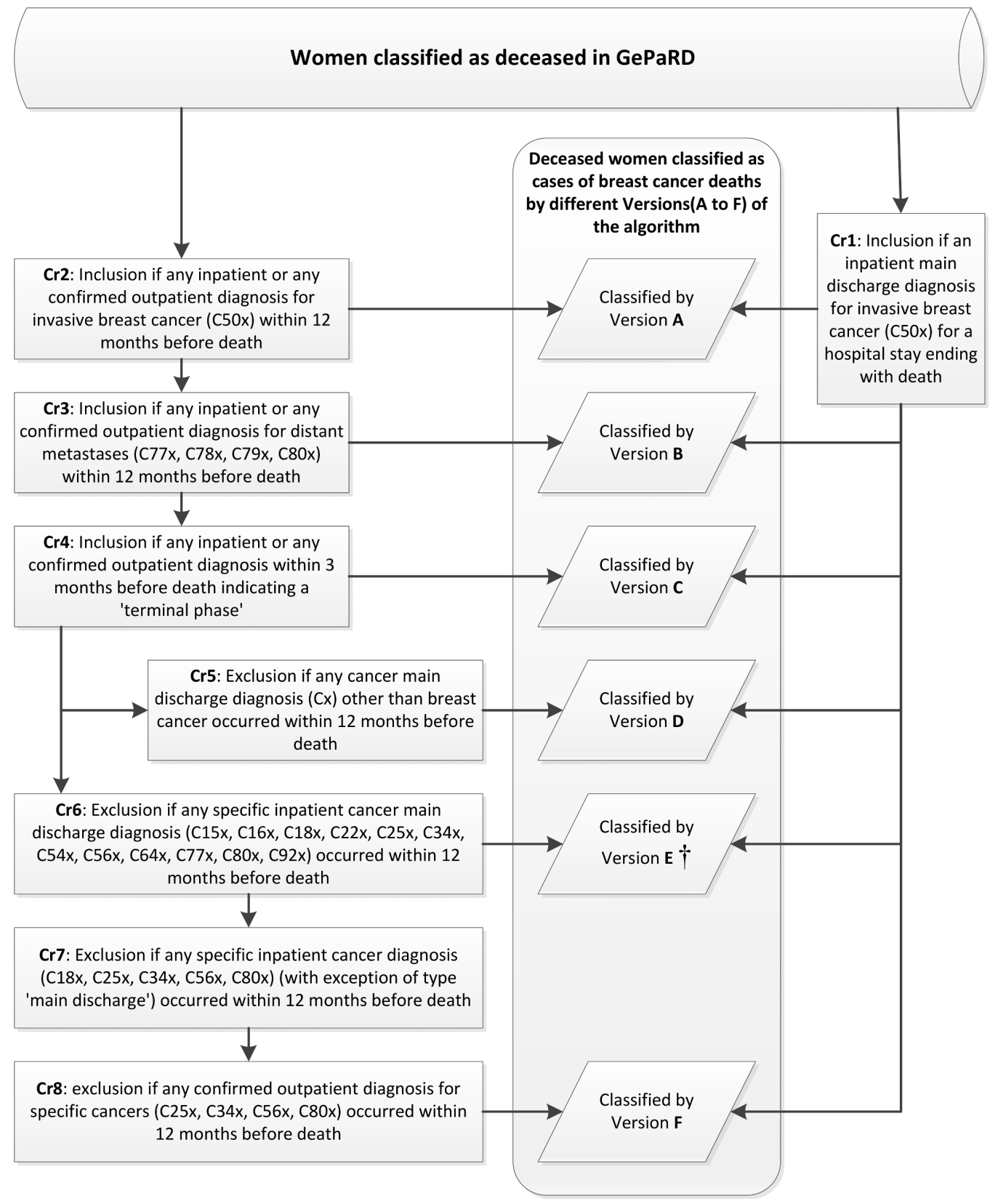

Figure 1 Data flow chart showing the criteria ( $\mathrm{Cr} 1-\mathrm{Cr} 8)$ and decision paths used in the different algorithm versions (A-F) to identify potential cases of breast cancer death among deceased women in the German Pharmacoepidemiological Research Database (GePaRD); cases fulfilling $\mathrm{Cr} 1$ were selected in each algorithm version; cases selected via the left decision path had to fulfil all criteria depicted on the path to the respective algorithm version (eg, version $\mathrm{E}$, left path: $\mathrm{Cr} 2$ and $\mathrm{Cr} 3$ and $\mathrm{Cr} 4$ and Cr6); diagnosis codes listed for the criteria are given in ICD-10-GM format (only three digit ICD codes are shown). †Selected for further evaluation. ICD-10-GM, German modification of the International Classification of Diseases, 10th Revision. 
analyses into 10 disjunct equally sized test samples (similar to a cross-validation but without using training samples for the optimisation of algorithm parameters). For each test sample, we calculated sensitivity, specificity, negative predictive value (NPV) and positive predictive value (PPV) for each version of the algorithm, using the official CoD information from the CR-NRW as gold standard. The results of these test samples are presented as median values and the corresponding variances in boxplot graphs, with the aim of identifying an algorithm that exhibited the best measures of accordance. In contrast to the study population used by Gagnon et $a l^{3}{ }^{3}$ our study sample included a high proportion of women who had died from causes other than BC. Therefore, to avoid high numbers of false positives, we put special emphasis on a high PPV in the selection of the most promising algorithm version, maintaining comparatively high values for specificity and sensitivity. Measures of accordance stratified by age were also calculated for the algorithm version selected on the basis of these criteria.

For population-level analyses, annual age-specific $\mathrm{BC}$ mortality rates were calculated by using the number of $\mathrm{BC}$ deaths according to the selected algorithm version as the numerator and the female population of GePaRD ever insured in the respective calendar year as denominator. These algorithm-based BC mortality rates were then compared with the official rates for the total German population as provided by the $\mathrm{ZfKD}$.

For the BC mortality rates and the measures of accordance, corresponding 95\% CIs were calculated according to the methods recommended by Newcombe and Altman. ${ }^{8}$

All analyses were carried out with SAS V.9.3.

\section{RESULTS}

Validation of algorithm-based CoD on the individual level

The study subsample comprised 22413 deceased females, whose records were successfully linked with CR-NRW records and the official $\mathrm{CoD}$ information was added. The majority of this sample was aged $60-79$ years $(65.7 \%)$. BC was documented as the official CoD in $10.9 \%$ (table 1 ). Females with $\mathrm{BC}$ as the official CoD were younger than those dying from other diseases.

Algorithm A, which required at least one BC diagnosis during the twelve months before death, reached a sensitivity of $97.5 \%$ and a specificity of $93.0 \%$. The NPV was $99.7 \%$ and the PPV was $62.9 \%$ (table 2, figure 2). The additional requirement of documented distant metastases during the 12 months before death (algorithm B) led to a lower sensitivity (94.3\%), a higher specificity (95.5\%), a comparable NPV $(99.3 \%)$ and a higher PPV (72.4\%). The additional consideration of diagnoses indicating a terminal phase of the disease during the 3 months before death (algorithm C) resulted in only minor changes of the validity measures. The additional requirement of the absence of other cancer main discharge diagnoses during the 12 months before death (algorithm D) led to a further reduction of the sensitivity $(89.9 \%)$ and the NPV $(98.8 \%)$ as well as a further increase of the specificity $(97.6 \%)$ and the PPV $(82.2 \%)$. Relaxing this criterion by considering main discharge diagnoses only for those cancer types which were the most frequent cancer CoDs among the false positives classified by algorithm $\mathrm{C}$ only slightly changed the quality measures (algorithm E). As the $\mathrm{CoD}$ 'cancer other than BC' was still frequent among the false positives of algorithm $\mathrm{E}$, we expanded

Table 1 Official cause of death (breast cancer; other causes) among women of the study population deceased between 2006 and 2013 in North Rhine Westphalia, by age group

Official cause of death

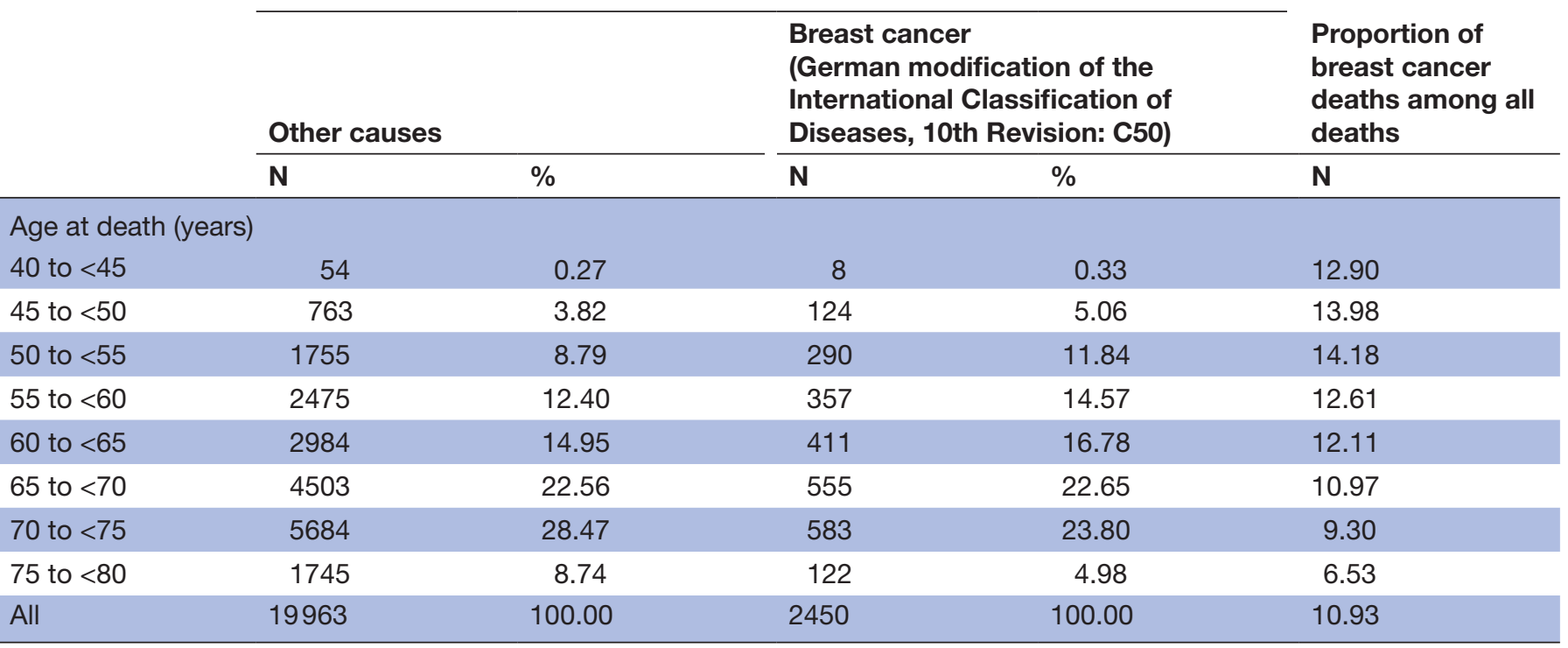

Records of the epidemiological cancer registry of North Rhine Westphalia. 
Table 2 Numbers and derived measures of accordance between the official cause of death (CoD) as the 'gold standard' and the $\mathrm{CoD}$ classification based on different versions of the $\mathrm{CoD}$ algorithm for breast cancer deaths

Measures of accordance (\%):

Median resulting from 10 test-samples

\begin{tabular}{|c|c|c|c|c|c|}
\hline Algorithm version & $\begin{array}{l}\text { Criteria used in the } \\
\text { algorithm version }\end{array}$ & Sensitivity & Specificity & $\begin{array}{l}\text { Negative } \\
\text { predictive value }\end{array}$ & $\begin{array}{l}\text { Positive predictive } \\
\text { value }\end{array}$ \\
\hline (A) & $\mathrm{Cr} 1, \mathrm{Cr} 2$ & 97.5 & 93.0 & 99.7 & 62.9 \\
\hline (B) & $\mathrm{Cr} 1-\mathrm{Cr} 3$ & 94.3 & 95.5 & 99.3 & 72.4 \\
\hline (C) & $\mathrm{Cr} 1-\mathrm{Cr} 4$ & 94.1 & 95.5 & 99.3 & 72.5 \\
\hline (D) & $\mathrm{Cr} 1-\mathrm{Cr} 5$ & 89.9 & 97.6 & 98.8 & 82.2 \\
\hline (E) & Cr1-Cr4, Cr6 & 91.3 & 97.4 & 98.9 & 81.7 \\
\hline$(F)$ & $\mathrm{Cr} 1-\mathrm{Cr} 4, \mathrm{Cr} 6-\mathrm{Cr} 8$ & 66.2 & 98.4 & 96.0 & 83.8 \\
\hline
\end{tabular}

Female residents of North Rhine Westphalia included in the German Pharmacoepidemiological Research Database who died between 2006 and 2013.

${ }^{*}$ For more details see figure 1 and online supplementary data 2.

the exclusion criteria by also considering inpatient secondary and outpatient diagnoses (algorithm F). Although we restricted this approach to cancers with a high fatality rate and a high prevalence in the study population to reduce false negatives, the sensitivity decreased to $66.2 \%$ while the specificity $(98.4 \%)$, NPV $(96.0 \%)$ and PPV $(83.8 \%)$ changed only slightly when compared with the results of algorithm E.
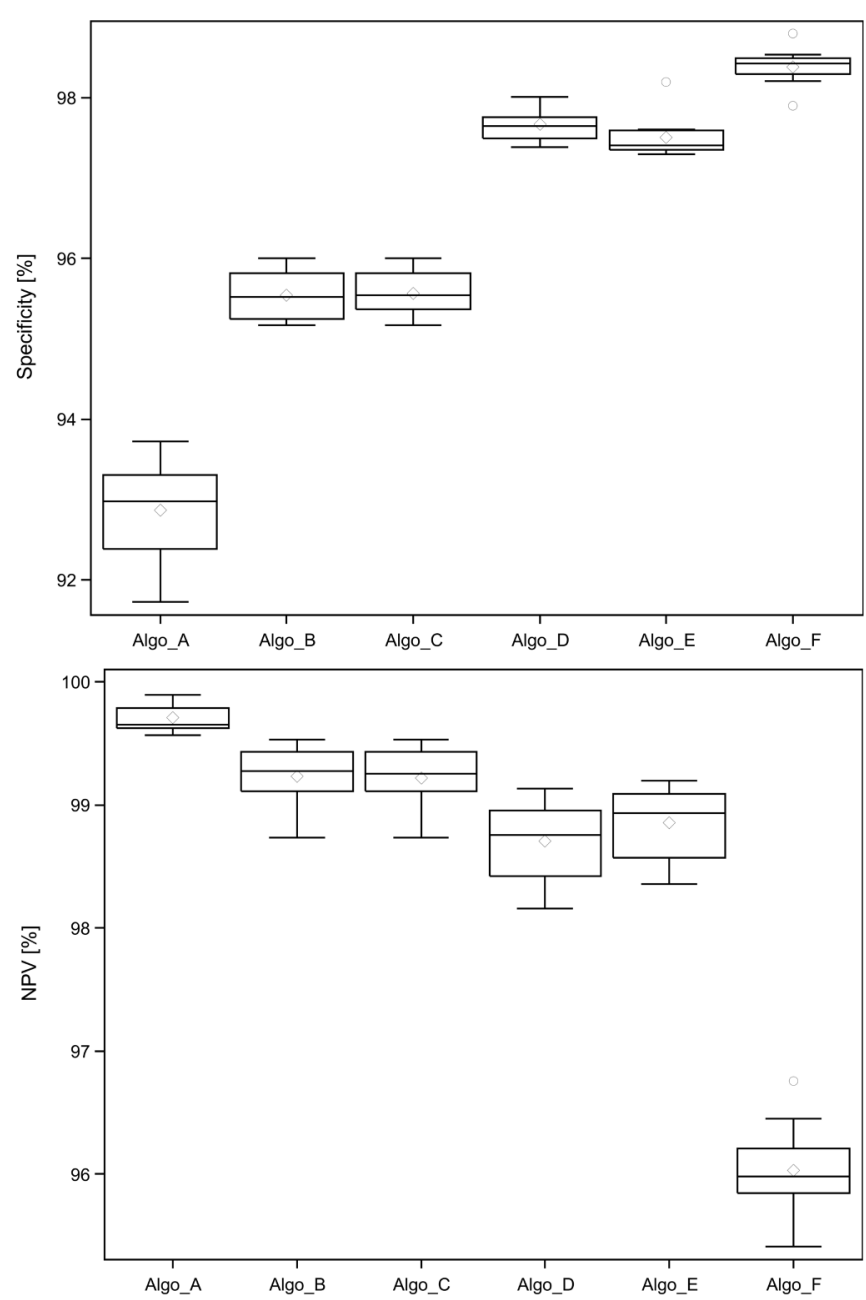

Figure 2 Boxplots for the results of the 10 disjunct equally sized test samples for sensitivity, specificity, negative predictive value (NPV) and positive predictive value (PPV) for the classification of six algorithm version (Algo_A to Algo_F) compared with the official cause of death. 
Table 3 Measures of accordance between the individual official cause of death (CoD) as the 'gold standard' and the CoD classification based on the breast cancer death algorithm (E), by age group

\begin{tabular}{|c|c|c|c|c|c|}
\hline \multirow[b]{2}{*}{ Age group (years) } & \multirow{2}{*}{$\begin{array}{l}\text { Breast cancer deaths } \\
\text { (N) as classified by } \\
\text { algorithm version (E) }\end{array}$} & \multicolumn{4}{|c|}{ Validity measures for accordance (\%) $(95 \%$ Cls) } \\
\hline & & Sensitivity & Specificity & $\begin{array}{l}\text { Negative predictive } \\
\text { value }\end{array}$ & $\begin{array}{l}\text { Positive } \\
\text { predictive value }\end{array}$ \\
\hline 40 to $<50$ & 156 & 96.2 (91.4 to 98.4 ) & 96.5 (94.9 to 97.5$)$ & 99.4 (98.5 to 99.7$)$ & 81.4 (74.6 to 86.7$)$ \\
\hline 50 to $<65$ & 1169 & 92.5 (90.8 to 94.0$)$ & 97.4 (97.0 to 97.7$)$ & $98.9(98.6$ to 99.1$)$ & 83.7 (81.5 to 85.8$)$ \\
\hline 65 to $<70$ & 611 & 91.2 (88.5 to 93.3 ) & 97.7 (97.2 to 98.1$)$ & 98.9 (98.5 to 99.2$)$ & 82.8 (79.6 to 85.6$)$ \\
\hline 70 to $<80$ & 788 & 87.0 (84.3 to 89.2 ) & 97.6 (97.3 to 98.0$)$ & 98.7 (98.5 to 99.0$)$ & 77.8 (74.8 to 80.6 ) \\
\hline
\end{tabular}

Among the versions with the highest values for specificity and PPV (D with $97.6 \%$ and $82.2 \%$, E with $97.4 \%$ and $81.7 \%$, F with $98.4 \%$ and $83.8 \%$ ), we selected algorithm $\mathrm{E}$ for the further evaluation as it offered the highest value for sensitivity $(91.3 \%$ vs $89.9 \%$ and $66.2 \%)$.

For algorithm E, sensitivity decreased with increasing age (from $96.2 \%$ in age group $40-50$ years to $87.0 \%$ in age group 70-80years) and the PPV was lowest in the highest age group $(77.8 \%$ in age group $70-80$ years vs $81.4 \%, 83.7 \%$ and $82.8 \%$ in the other age groups) while only marginal changes with age occurred for the specificity (between $96.5 \%$ and $97.7 \%$ ) and the NPV (between $98.7 \%$ and $99.4 \%$ ) (table 3 ).

\section{Comparison of algorithm-based and official BC mortality rates at population level}

The study samples exemplarily used for the calculation of the algorithm-based BC mortality rates in 2007, 2010 and 2012 comprised $n=7257$ 975, $n=7540664$ and $\mathrm{n}=7825758$ females, respectively. It included $\mathrm{n}=47763$, $\mathrm{n}=55013$ and $\mathrm{n}=60506$ deceased women of which $\mathrm{n}=2709$, $n=2959$ and $n=3141$, respectively, were classified as BC deaths by algorithm $\mathrm{E}$. The $\mathrm{BC}$ mortality rates based on algorithm $\mathrm{E}$ agreed well with the data from the ZfKD in all age categories except for the highest age group ( $\geq 85$ years) where the algorithm-based rates were $25 \%-30 \%$ lower than the national rates from the ZfKD (figure 3). This difference was significant in all study years.

\section{DISCUSSION}

In this study, we demonstrated the feasibility of a health claims data-based algorithm for the identification of BC deaths. Using German SHI claims data, we adapted the algorithm presented by Gagnon et $a \hat{l}$ for Canadian administrative hospital data by also including outpatient diagnoses and adding further criteria to optimise the performance of the algorithm. We validated the different algorithm versions by direct comparison with the official CoD on the individual level as well as by indirect comparison with official data on the population level.

Based on a sample of more than 22000 deceased women from our data source, the most promising algorithm comprised data on the presence of $\mathrm{BC}$, metastases, diagnoses indicating terminal illness and the absence of other tumours with a high case fatality rate as potentially competing CoDs. These individual criteria were applied within the quarter (terminal illness) or the year prior to death. The finally selected algorithm showed high

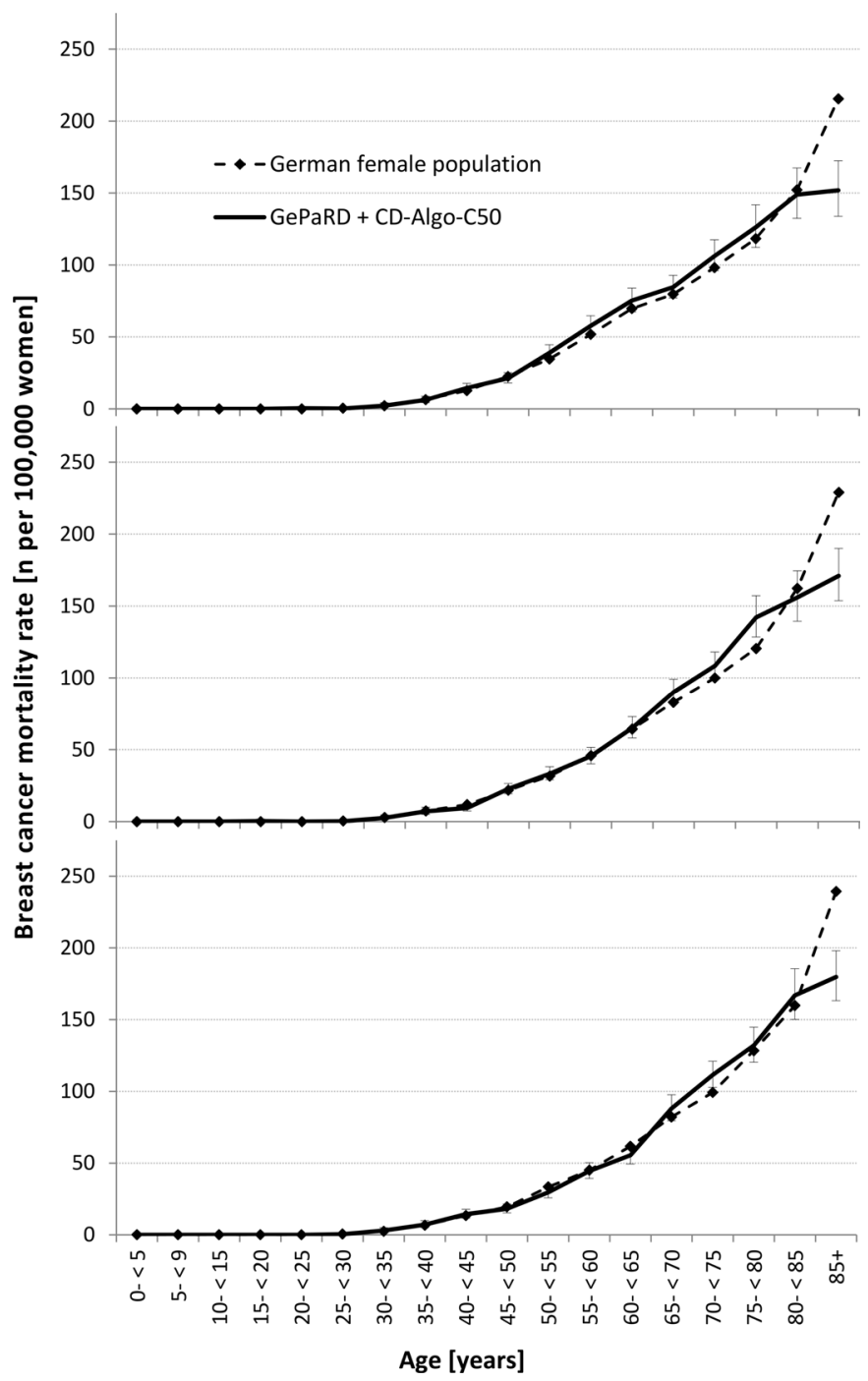

Figure 3 Official age-specific breast cancer mortality rates for German women in 2007, 2010 and 2012 as well as corresponding rates and $95 \%$ confidence limits (vertical bars) calculated using the female population included in the German Pharmacoepidemiological Research Database $(\mathrm{GePaRD})$ in these years, respectively, and the classification of deceased women resulting from the application of the cause of death algorithm version (E) for breast cancer. 
specificity and NPVs and represents a compromise in terms of a reasonably high sensitivity and a PPV that is not too low.

On the population level, the application of this algorithm resulted in a good agreement of algorithm-based age-specific BC mortality rates in the GePaRD sample with general population rates. However, the predictive performance (expressed as sensitivity and PPV) of the algorithm was better for deaths below the age of 70 than for older ages. Especially for the age group of 85 years and older, we saw a marked underestimation of the official rate by the algorithm-based rates.

One reason for this finding could be that the intensity of the search for metastases might be lower in very old patients with cancer compared with younger ones as elderly patients are less likely to receive additional cancer treatment. ${ }^{910}$ Consequently, as the algorithm requires identifying metastasis diagnoses for the classification of $\mathrm{BC}$ deaths, the rate of false negative decisions by the algorithm would be higher among the elderly leading to a more pronounced underestimation of the BC mortality rate in this age group. Indeed, among cases with an official CoD 'BC' which were misclassified by the algorithm, the rate of those misclassified due to missing metastasis diagnoses increased with age (results not shown), which in part explains the slightly lower PPV for the age group 70 years and older compared with the younger age groups. As the sample enriched with the individual official CoD excluded subjects aged 80 years or older, we were not able to examine reasons for the underestimation on the individual level in more detail.

We explored the validity of several algorithm versions, which differed from the approach presented by Gagnon et $a l^{3}{ }^{3}$ For this, we used an approach with 10 test samples to explore the variance of the estimates of the measures of accordance. Certain changes between two algorithm versions (version $\mathrm{B}$ vs $\mathrm{C}$ and version $\mathrm{D}$ vs $\mathrm{E}$ ) led only to minor changes of the measures of accordance. In extension to version $\mathrm{B}$, version $\mathrm{C}$ required the presence of at least one condition indicating terminal illness. ${ }^{3}$ As the corresponding list of diagnoses is rather long, the inclusion of ambulant diagnoses in addition to inpatient diagnoses (in contrast to Gagnon et al) might have increased the probability of such diagnoses being present in an individual which could have weakened the discrimination of this criterion. In contrast to algorithm version $\mathrm{D}$, version $\mathrm{E}$ did not exclude all individuals with any inpatient cancer main discharge diagnoses of cancer (criterion Cr5) but only those with such diagnoses for specific tumours (criterion Cr6). This restriction was intended to reduce false negatives by focusing on tumours with a higher fatality rate. However, this characteristic could be associated with a higher probability of such diagnoses occurring as a main discharge diagnosis compared with diagnoses with a lower fatality rate that would reduce the differences in discrimination between criterion Cr5 and criterion Cr6. The setting of our algorithm $\mathrm{C}$ was the version most similar to the algorithm used by these authors, however, with the major difference that we also considered outpatient diagnoses. Compared with their results (sensitivity 95\%, specificity $89 \%$, PPV 98\%, NPV $77 \%$ ) which were based on a sample of only 119 deceased women, our algorithm $\mathrm{C}$ showed a higher specificity and NPV, a similar sensitivity but a lower PPV. Adding further criteria lowered the rate of false positives but led to a decline in sensitivity (our algorithm versions D-F).

Of note, the comparison of the validity measures obtained in the two studies is constrained by the fact that, contrary to our approach of using the official $\mathrm{CoD}$ for validation, Gagnon et al relied on the decision of a palliative care specialist who reviewed the entire hospital medical chart to determine whether a study subject had died of $\mathrm{BC}$ or not. Furthermore, Gagnon et a $\hat{l}^{3}$ derived their algorithm from a sample of women with BC in need of end-of-life cancer care, while our approach intended to identify the outcome 'BC death' among deceased women in an epidemiological follow-up study. Therefore, the two studies may have prioritised different criteria to select the 'best-fitting' algorithm. It should further be noted that Canadian study setting, characterised by only a small proportion $(16 \%)$ of women not dying of $\mathrm{BC}$, that is, a high a priori probability of $\mathrm{BC}$ death, facilitated the generation of validity measures with a combination of high specificity, high PPV and low NPV. By contrast, our study sample comprised a proportion of $89 \%$ of women not dying of $\mathrm{BC}$, that is, a low a priori probability of $\mathrm{BC}$ death, which more likely leads to results with combinations of high sensitivity, low PPV and high NPV. Another notable difference in the study setting was that we did not restrict our sample to women dying in hospital (about $50 \%$ of all cases included in our study). Therefore, comparisons between the study of Gagnon et al and our study should only be cautiously invoked. However, it is generally accepted that a high PPV is important to minimise bias in relative effect estimates, that is, a high PPV will be important when the algorithm is used for outcome definition in future studies on risk factors of BC death or the evaluation of screening measures.

\section{Limitations}

Some general limitations have to be considered when interpreting our results. First, this study shows that it appears principally feasible to adapt published algorithms to the specific characteristics and requirements of another data setting such as a claims database in another country. Of note, $87 \%$ of the German population are insured by SHIs all of which routinely collect claims data on the same structured data basis as GePaRD and the algorithm presented here is most likely also applicable to data from other German SHIs. In theory, the algorithm could even be transferred to other countries because the three-digit diagnoses used (figure 1) are the same in the international version of ICD-10. However, the purpose of collecting health data and coding practices may be different and this, in turn, could influence the informative quality (specificity) of the diagnoses included. Further, 
different profoundness and details in the medical data at hand (eg, tumour staging, disease severity, laboratory measures) may necessitate modifications of the algorithm and subsequently also additional evaluation.

Second, our reference, the official CoD, is not in perfect agreement with the true underlying $\mathrm{CoD}$. There is an acknowledged degree of uncertainty in the abstraction of the CoD from death certificates with some inter-rater variability. ${ }^{112}$ For example, the medical examiner may occasionally not be aware of the full medical history omitting relevant concomitant disease data. ${ }^{13}{ }^{14}$ In contrast, the algorithm in our study was based on all medical diagnoses recorded in the year before death. Thus, using the official $\mathrm{CoD}$ as a gold standard for the validation of the algorithm was a pragmatic choice in this study. Therefore, achieving complete agreement between the official and the algorithm-based CoD 'BC' was not to be expected.

Third, the record linkage procedure applied to add the official $\mathrm{CoD}$ to the GePaRD data was based on a probabilistic method using pseudonymised data. The linkage method exhibited very low error rates in an independent validation study. ${ }^{15}$ Additionally, the matches resulting from the probabilistic linkage used in this study were verified by a second, but different linkage method. ${ }^{7}$ Mismatches in the linkage procedure with subsequent misclassification, which would result in an underestimation of the performance of the algorithm versions examined in this study, are thus expected to be low. Further, only $5.28 \%$ of the records of the original linkage sample had no successful link with CR-NRW data. The cases that could not be linked may be explained by data errors at the CR-NRW or in the core data of the SHIs concerning the personal identifiers used for the linkage. ${ }^{7}$ These data errors (mostly entry errors or various spellings of complex names) are unlikely to be associated with the CoD. The proportion of $10.9 \%$ with an official CoD 'BC' among those with a successful link corresponds closely to national data of the Federal Statistical Office ${ }^{16}$ in the observed age range. Thus, substantial biases introduced by the linkage procedure appear unlikely.

Fourth, although the age-specific BC mortality rates produced with algorithm $\mathrm{E}$ and the official $\mathrm{BC}$ mortality rates provided by the ZfKD showed hardly any differences one needs to keep in mind differences between the underlying study populations. While the latter are representing the general population of Germany, three of the four SHIs contributing data to $\mathrm{GePaRD}$ comprise relatively high proportions of insured persons with a better educational and economic position. ${ }^{17}$ Social position is generally associated with morbidity and mortality risk, ${ }^{18}$ and meta-analyses indicate increased $\mathrm{BC}$ incidence rates in women with higher socioeconomic status, ${ }^{19}{ }^{20}$ while the evidence regarding differences in BC mortality is heterogeneous. ${ }^{19}$ Therefore, it is not clear whether the selection of SHIs represented in the database could have had an effect on the algorithm-based BC mortality rate. Additionally, the official mortality rates are based on the monocausal documentation of the underlying CoD.
Thus, if two different cancer diseases are documented by the medical examiner on the death certificate, the official $\mathrm{CoD}$ is coded as 'malignant neoplasms of independent (primary) multiple sites' (ICD-10-GM: C97). Of note, for only $0.6 \%$ of our sample $(n=67)$ was $\mathrm{C} 97$ the official $\mathrm{CoD}$ but $\mathrm{n}=10$ of them were classified as $\mathrm{BC}$ deaths by algorithm $\mathrm{E}$. This, however, limits the comparability of the algorithm-based mortality rates with official data only to a small extent.

Fifth, not all of the criteria defined for the different algorithm versions were defined independently from the data used for the evaluation of the algorithm performance. Three of eight criteria were defined after additional considerations following a descriptive analysis of the official causes of death of those selected as false positives results with another algorithm version which was based on a priori set criteria. Although we did not apply typical machine learning for the algorithm versions in our study and we did not directly analyse the diagnosis entries used by the algorithm to classify deceased women into 'BC deaths' and 'non-BC deaths', our procedure might have led to some overoptimistic results for two of the six tested algorithm versions. On the other hand, the boxplots of the results of the test samples showed only small variances for the calculated accordance measures of the test samples that indicates rather a robust algorithm.

\section{CONCLUSION}

Our study showed that the algorithm-based classification of $\mathrm{BC}$ as $\mathrm{CoD}$, as adapted from a recently published Canadian algorithm, is feasible with German claims-based data (with the possible exemption of the age group $85+$ years). This may indicate that such algorithms could also be adapted to healthcare databases in other countries. If individual linkage with official CoD is not possible, the algorithm is a useful tool that can be applied in epidemiological studies using BC mortality as an endpoint, for example, in the context of monitoring and evaluating routine MSPs. Given that in Germany, about $90 \%$ of the total female population are members of an SHI the algorithm could be used to investigate $\mathrm{BC}$ deaths in the vast majority of the German female population. However, given the variability observed with different algorithm versions, the transferability of the adapted algorithm to other data sources will certainly require further specific adaptations and additional validation.

\section{Author affiliations}

${ }^{1}$ Clinical Epidemiology, Leibniz Institute for Prevention Research and Epidemiology BIPS, Bremen, Germany

${ }^{2}$ Health Services Research, IGES Institut GmbH, Berlin, Germany

${ }^{3}$ High-Profile Research Area Health Sciences, University of Bremen, Bremen,

Germany

${ }^{4}$ Institute of Epidemiology and Social Medicine, Westfälische Wilhelms-Universität Münster, Münster, Germany

${ }^{5}$ State Cancer Registry North Rhine Westphalia, Münster, Germany

${ }^{6}$ SOCIUM Research Center on Inequality and Social Policy, University of Bremen, Bremen, Germany 
Acknowledgements We would like to thank the AOK Bremen/Bremerhaven, the hkk Krankenkasse, the Techniker Krankenkasse (TK) and the DAK-Gesundheit, which provided data for this study. We also thank the Techniker Krankenkasse (TK) and the DAK-Gesundheit for their intensive support. The study presented here was conducted in the framework of a feasibility study for the evaluation of breast cancer-related mortality in the German mammography screening programme, which was funded by the Federal Office for Radiation Protection (Bundesamt für Strahlenschutz), the Federal Ministry for Environment, Nature Conservation, Building and Nuclear Safety (BMUB), the Federal Ministry of Health (BMG) and the Kooperationsgemeinschaft Mammographie under grant no. UFOPLAN 3610 S40002 and $3614 S 40002$. The publication of this article was funded by the 0pen Access Fund of the Leibniz Association.

Contributors IL, $\mathrm{CO}, \mathrm{UH}, \mathrm{HWH}$ and $\mathrm{HZ}$ conceived the study and planned the design. IL and UH obtained the permission from SHIs and administrative authorities to use the claims data for this study. IL and HWH managed the record linkage between claims data and epidemiological cancer registry. IL performed the statistical analyses. IL and CO wrote the first draft of the manuscript. UH, HWH, JC and HZ provided supervision and critical review of the manuscript. All authors contributed to and have approved the final manuscript.

Funding The present study was funded by the Federal Office for Radiation Protection (Bundesamt für Strahlenschutz) of the Federal Ministry for Environment, Nature Conservation and Nuclear Safety (BMU), the Federal Ministry of Health (BMG) and the Kooperationsgemeinschaft Mammographie under grant no UFOPLAN 3610 S40002 and 3614 S40002.

Competing interests None declared.

Patient consent for publication Not required.

Ethics approval The utilisation of SHI data for scientific research is regulated by the Code of Social Law in Germany (SGB X). All four involved SHIs, the Federal Social Insurance Authority (as the responsible authority of the three nationwideoperating SHIs) and the regional Senator for Science, Health and Consumer Protection (for the one regional SHI) approved the use of the data for this study.

Provenance and peer review Not commissioned; externally peer reviewed.

Data sharing statement In accordance with German data protection regulations, access to the data of the German Pharmacoepidemiological Database must not be given to third parties. Furthermore, as we are not the owners of the data we are not legally entitled to grant access to the data.

Open access This is an open access article distributed in accordance with the Creative Commons Attribution Non Commercial (CC BY-NC 4.0) license, which permits others to distribute, remix, adapt, build upon this work non-commercially, and license their derivative works on different terms, provided the original work is properly cited, appropriate credit is given, any changes made indicated, and the use is non-commercial. See: http://creativecommons.org/licenses/by-nc/4.0/.

\section{REFERENCES}

1. Malek D, Kääb-Sanyal V. Implementation of the German Mammography Screening Program (German MSP) and First Results for Initial Examinations, 2005-2009. Breast Care 2016;11:183-7.

2. Fuhs $\mathrm{A}$, Bartholomäus $\mathrm{S}$, Heidinger $\mathrm{O}$, et al. Evaluation of effects of the Mammography-Screening-Program on breast cancer mortality: feasibility study fort he record linkage of different data sources in North Rhine-Westphalia [Evaluation der Auswirkungen des Mammographie-Screening-Programms auf die Brustkrebsmortalität: Machbarkeitsstudie zur Verknüpfung verschiedener Datenquellen in Nordrhein-Westfalen]. Bundesgesundheitsb/ 2014;57:60-7.
3. Gagnon B, Mayo NE, Laurin C, et al. Identification in administrative databases of women dying of breast cancer. J Clin Oncol 2006;24:856-62.

4. Ohlmeier C, Langner I, Hillebrand K, et al. Mortality in the German Pharmacoepidemiological Research Database (GePaRD) compared to national data in Germany: results from a validation study. BMC Public Health 2015;15:570.

5. Garbe E, Mikolajczyk RT, Banaschewski T, et al. Drug treatment patterns of attention-deficit/hyperactivity disorder in children and adolescents in Germany: results from a large population-based cohort study. J Child Adolesc Psychopharmacol 2012;22:452-8.

6. Pigeot I, Ahrens W. Establishment of a pharmacoepidemiological database in Germany: methodological potential, scientific value and practical limitations. Pharmacoepidemiol Drug Saf 2008;17:215-23.

7. Langner I, Krieg V, Heidinger O, et al. [Enrichment of Claims Data with Official Causes of Death Using a Record Linkage with the Epidemiological Cancer Registry of North Rhine-Westphalia: Feasibility Study and Comparison of Procedures]. Gesundheitswesen 2018.

8. Newcombe RG, Altman DG, et alProportions and their differences. In: Altman DG, Machin D, Bryant TN, Gardner MJ, . eds. Statistics with confidence. 2nd ed. Bristol: JW Arrowsmith Ltd, 2001:45-56.

9. Chawla N, Yabroff KR, Mariotto A, et al. Limited validity of diagnosis codes in Medicare claims for identifying cancer metastases and inferring stage. Ann Epidemiol 2014;24:666-72.

10. Warren JL, Mariotto A, Melbert D, et al. Sensitivity of Medicare Claims to Identify Cancer Recurrence in Elderly Colorectal and Breast Cancer Patients. Med Care 2016;54:e47-e54.

11. Jahn I, Jöckel KH, Bocter N, et al. Studie zur Verbesserung der Validität und Reliabilität der amtlichen Todesursachenstatistik. Baden-Baden: Nomos 1995:230.

12. Giersiepen K, Greiser E. [Coding of cause of death for mortality statistics-a comparison with results of coding by various statistical offices of West Germany and West Berlin]. Offentl Gesundheitswes 1989;51:40-7.

13. Schubert-Fritschle G, Eckel R, Eisenmenger W, et al. Quality of indications on death certificates- Is the cause of death statistic concerning cancer better than its reputation? [Qualität der Angaben von Todesbescheinigungen - Ist die Todesursachenstatistik zu Krebserkrankungen besser als ihr Ruf?]. Dtsch Arzteb/ 2002;99:50-5.

14. Schröder AS, Wilmes S, Sehner S, et al. Post-mortem external examination: competence, education and accuracy of general practitioners in a metropolitan area. Int $J$ Legal Med 2017;131:1701-6.

15. Schmidtmann I, Sariyar M, Borg A, et al. Quality of record linkage in a highly automated cancer registry that relies on encrypted identity data. GMS Med Inform Biom Epidemiol 2016;12.

16. Federal Statistical Office. Mortality Statistics. $2019 \mathrm{https} / / / \mathrm{www}-$ genesis.destatis.de/genesis/online/data;sid=7A797275F348011F 6C4D45595BCE75CD.GO_1_3?operation=abruftabelleAbrufen\& selectionname $=23211-0004$ \&levelindex $=0$ \&levelid $=1550570635876 \&$ index $=4$ (Accessed 19 Feb 2019).

17. Hoffmann F, Icks A. [Structural differences between health insurance funds and their impact on health services research: results from the Bertelsmann Health-Care Monitor]. Gesundheitswesen 2012;74:291-7.

18. Mackenbach JP, Kunst AE, Cavelaars AE, et al. Socioeconomic inequalities in morbidity and mortality in western Europe. The EU Working Group on Socioeconomic Inequalities in Health. Lancet 1997;349:1655-9.

19. Akinyemiju TF, Genkinger JM, Farhat M, et al. Residential environment and breast cancer incidence and mortality: a systematic review and meta-analysis. BMC Cancer 2015;15:191.

20. Lundqvist A, Andersson E, Ahlberg I, et al. Socioeconomic inequalities in breast cancer incidence and mortality in Europe-a systematic review and meta-analysis. Eur J Public Health 2016;26:804-13. 